\title{
THREE-DIMENSIONAL RECONSTRUCTION OF ARTERIAL-LYMPHATIC VASCULAR COMPLEX (VESSEL WITHIN THE VESSEL) IN BREAST TISSUE
}

\author{
Ivan N. Ivanov, \\ Savelina L. Popovska, \\ Emilian A. Ivanov', \\ Sergey D. Iliev, \\ Pencho T. Tonchev ${ }^{2}$ \\ Department of Pathology, \\ Medical University - Pleven \\ ${ }^{1}$ Department of Anatomy, Histology \\ and Cytology and Biology. \\ Medical University - Pleven \\ ${ }^{2}$ Department of Propaedeutics of \\ Surgical Diseases, \\ University Hospital - Pleven
}

\author{
Corresponding Author: \\ Ivan N. Ivanov \\ Department of Pathology \\ Medical University - Pleven \\ 1, St. Kliment Ohridski str. \\ Pleven, 5800 \\ Bulgaria \\ e-mail: posledenzalez@gmail.com
}

Received: December 18, 2012

Revision received: March 01, 2013

Accepted: June 26, 2013

\section{Summary}

The phenomenon "vessels within vessels" was initially described by Merchant $\mathrm{S}$ et al. as arteries found freefloating inside the lumen of veins. We have described another version of the "vessels within vessels"complex, composed of arteries found inside the lumen of lymphatic vessels. The purpose of the present study was to describe the structure of the lympho-arterial vascular complex in a breast tissue specimen from a male subject using threedimensional tissue reconstruction. A histological specimen from a 64 year-old male subject diagnosed with gynecomastia was used. The tissue sample was sectioned in a multi-step manner. An overall of approximately $150 \mu \mathrm{m}$ thick tissue material was sampled. Immunostaining with anti-CD34 antibody and anti-podoplanin antibody was performed. Three-dimensional reconstruction of the vessel within the vessel structure was performed with "Reconstruct" software When the reconstruction of the breast parenchyma was revealed as a 3D image, it became apparent that the arterial vessel was situated inside the lymphatic vessel and could be followed along the entire length of the vascular segment studied. We have proved that these vascular complexes are not artificial phenomenon and do exist. The function of the vascular complexes is still uncertain, and is probably related to lymph propulsions in the initial collector vessels.

Key words: lymphatic-arterial vascular complex; male breast; 3D tissue reconstruction

\section{Introduction}

The phenomenon "vessels within vessels" was described by Merchant et al. ten years ago as a strange histological finding within the myometrium - "... a peculiar vascular architecture in the myometrium wherein arteries are found free-floating within cleft-like spaces". The latter were identified as venous channels. Such "vessel within the vessel" complexes were also described in the breast [1] .

These vascular complexes were considered to be pathogenetically related to menorrhagia [1] and eventually to the development of intravenous leiomyomatosis $[1,2]$. However, the morphology of 
the phenomenon "vessels within vessels" is presently poorly understood.

Recently we have described the presence of a vascular complex, composed of pre-collecting lymphatic vessels surrounding a small-calibre artery/arteriole in the female breast [3]. The presence of vessels within vessels complexes has, so far, not been studied in breast tissue specimens from male subjects. Our observations on breast specimens from male patients showed the presence of vascular complexes, composed of pre-collecting lymphatic vessels surrounding a small-calibre artery /arteriole (unpublished data). The vascular complexes found in males had even more prominent and distinct morphology, as compared to the morphology seen in the breast of female subjects.

The purpose of the present study was to describe the structure of the lympho-arterial vascular complex in a breast tissue specimen from a male subject using three-dimensional tissue reconstruction.

\section{Materials and Methods}

A histological specimen from a 64 year-old male subject diagnosed with gynecomastia was used. The tissue sample was sectioned for diagnostic purposes in a multi-step manner because of suspicious focal florid epithelial overgrowth in ducts, that later turned out to be benign. On the other hand, the series revealed a traceable vessel within the vessel complex.

The multi-step sectioning of the specimen was performed in the following manner. First, a $4 \mu \mathrm{m}$ thick section was made and mounted on a histological slide, and $20 \mu \mathrm{m}$ of tissue were then cleared to expose underlying structures. Another $4 \mu \mathrm{m}$ thick section was made and mounted on a histological slide. That algorithm was followed until seven slides were prepared. The first 5 slides were stained with H\&E (routine staining). The benign nature of the lesion was confirmed in the first 4 slides, and the remaining two slides were immunostained with CD34 and D2-40. An overall of approximately $150 \mu \mathrm{m}$ thick tissue material was sampled.

\section{Immunohistochemistry}

The prepared $4 \mathrm{~m}$ thick tissue samples (slides six and seven) were deparafinised in xylene, and rehydrated with ethanol in a series of decreasing concentrations. Immunostaining with anti-CD34 antibody clone 1A4 (Dako) and anti-podoplanin antibody clone D2-40 (Dako) was performed in accordance with standard laboratory protocols. Staining was interpreted in accordance with criteria set by the manufacturer.

\section{Three-dimensional reconstruction}

Initially, the vessel within the vessel structure was identified on every slide. Then the consecutive sections of the "vessel within the vessel" structure were photographed using an Olympus BX 40 microscope equipped with Olympus C 5050 ZOOM camera. The digital images (in JPEG file format) were consecutively numbered and stored on a CD.

Three-dimensional reconstruction of the "vessel within the vessel structure" was performed with "Reconstruct" software [4].

The process of $3 \mathrm{D}$ reconstruction was performed in several main steps. First, a new Series file [*.ser] was created. Then the digital images of the histological slides were imported in the program, the program was calibrated and the images were aligned. The draw freehand tool was used to outline the lymphatic vessel in yellow, and the blood vessel in red. Finally, all traced objects were selected from a list (option "Object" from the toolbar of Reconstruct) and a 3D scene was created. The 3D scene, which in our case was representing the tissue fragments from the two vessels, was saved in its most illustrative twodimensional projection as a JPEG file.

\section{Results}

Two examples of arteries situated within the lumen of pre-collector lymphatic vessels were found within the studied tissue (Figure 1 sections A, B, C). artery situated within the lumen of vein was found.

Three main positions of the artery within the pre-collecting lymphatic vessel were detected: an artery prominent within the lumen of the lymphatic vessel as a vascular-stromal protrusion (Figure 2 section A); an artery, free-floating within the lumen of the lymphatic vessel (Figure 2 section B) and an artery floating within the lumen of the lymphatic vessel, anchored to the wall of the lymphatic vessel by a fine connective tissue strand, lined with lymphatic endothelium (Figure 2 section C).

When the reconstruction of the breast parenchyma was revealed as a 3D image, it became apparent that the arterial vessel that was situated inside the lymphatic vessel could be 


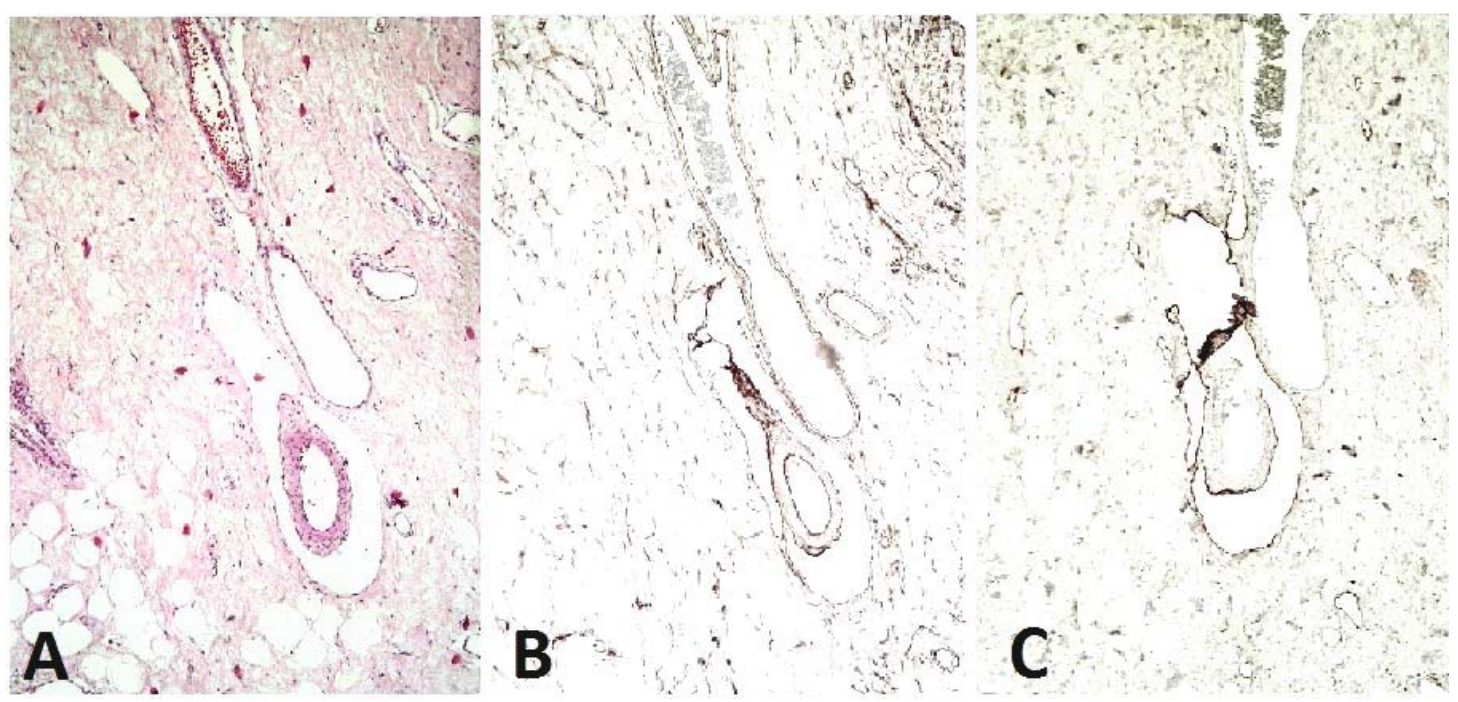

Figure 1. A) Vessel within the vessel phenomenon. Artery situated within the lumen of pre-collector lymphatic vessel- large vessel, lined with endothelium, surrounded by incomplete circular layer of smooth muscle cells - H\&E, 100x; 1. B) CD34 staining, demonstrating positive reaction in stromal fibroblasts and blood vessels endothelium. The endothelium of the internal (arterial vessel) is positive for CD34 - CD34, 100x; C) Only lymphatic endothelium was positive for D2-40 staining. The external vessel from the vessel within the vessel complex was found to be lined with D2-40 positive endothelium. D2-40, 100x

followed along the entire length of the vascular segment studied $(\sim 150 \mu \mathrm{m})$. The artery inside the lymphatic vessel was not free-floating inside the lumen of the lymphatic vessel. It was stabilized by separate strands of connective tissue, covered with endothelium (Figure 2 section $\mathrm{C}$ - arrow). A vascular-stromal protrusion was visible at the probable terminal end of the complex, where the artery entered or left the lymphatic vessel. It was a relatively thick stem, surrounding the arterial vessel, and was part of the normal breast stroma that was composed of CD34 positive fibroblasts. This connective tissue stem supported the vessel, which was located partly inside the vascular lumen.

The vessel observed within the vessel complex (lymho-arterial vascular complex) in the context of the breast blood and lymph circulation is presented on Figure 3.

\section{Discussion}

This study is the first to describe the presence of vascular-vascular complexes, composed of precollecting lymphatic vessels surrounding a smallcalibre artery/arteriole in a male breast. On the other hand, the classical vessel-within-the-vessel pattern, described by Merchant et al. [1] was not found in the tissues examined, but it was likely to exist.

The 3D reconstruction of a $150 \mu \mathrm{m}$ long segment of the vascular complex, composed of pre-collecting lymphatic vessel surrounding a small-calibre artery, proved the actual existence of that structure. The confirmation of the existence of the vascular complex, composed of pre-collecting lymphatic vessel surrounding a small-calibre artery raises questions about the functional role of this morphological unit.

The functions of the "vessel-within-thevessel" structure described are possibly related to lymph propulsion within the initial collecting lymphatic vessels and regulation of the normal lymph flow within the breast tissue. Those vessels (precollectors) do not possess a strong continuous smooth muscle layer $[5,6]$ and probably are unlikely to produce sufficient lymph propulsion on their own, when functional requirements are increased. There are no muscle contractions to support lymph flow in the breast, either. It is well known that lymph might be "pushed" by the pulsation of arteries adjacent to the lympho-vascular wall [7]. Logically, the optimal transmission of the impulse, created by the arterial pulsation, is achieved when the 


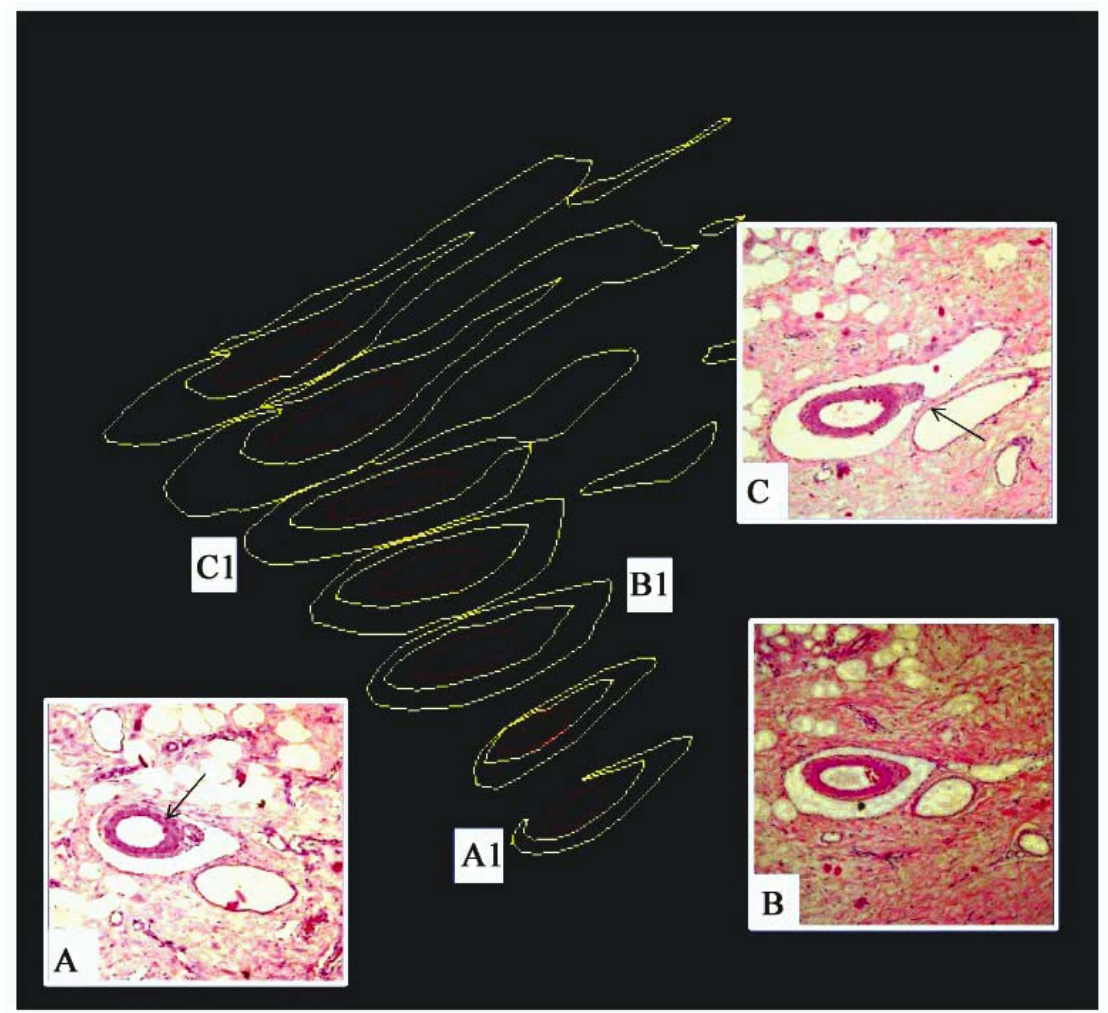

Figure 2. A two-dimensional projection of the studied segment of the vascular complex, composed of pre-collecting lymphatic vessel surrounding a small-calibre artery. On section A (corresponding to segment A1 of the 3D model) the artery is seen partly floating inside the pre-collecting lymphatic vessel. It appears like a protrusion which might be easily partly dislocated from the lumen of the lymphatic vessel. Section B (corresponding to segment B1 of the 3D model) demonstrates an artery free-floating within the lumen of the lymphatic vessel. The pulsations of the arterial wall might be transformed easily to the lymph inside the lymphatic vessel. Section $\mathbf{C}$ (corresponding to segment $\mathrm{C} 1$ of the 3D model) demonstrates artery floating within the lumen of the lymphatic vessel, anchored to the wall of the lymphatic vessel by fine connective tissue strand, lined with lymphatic endothelium. Those were seen in two slides from this series

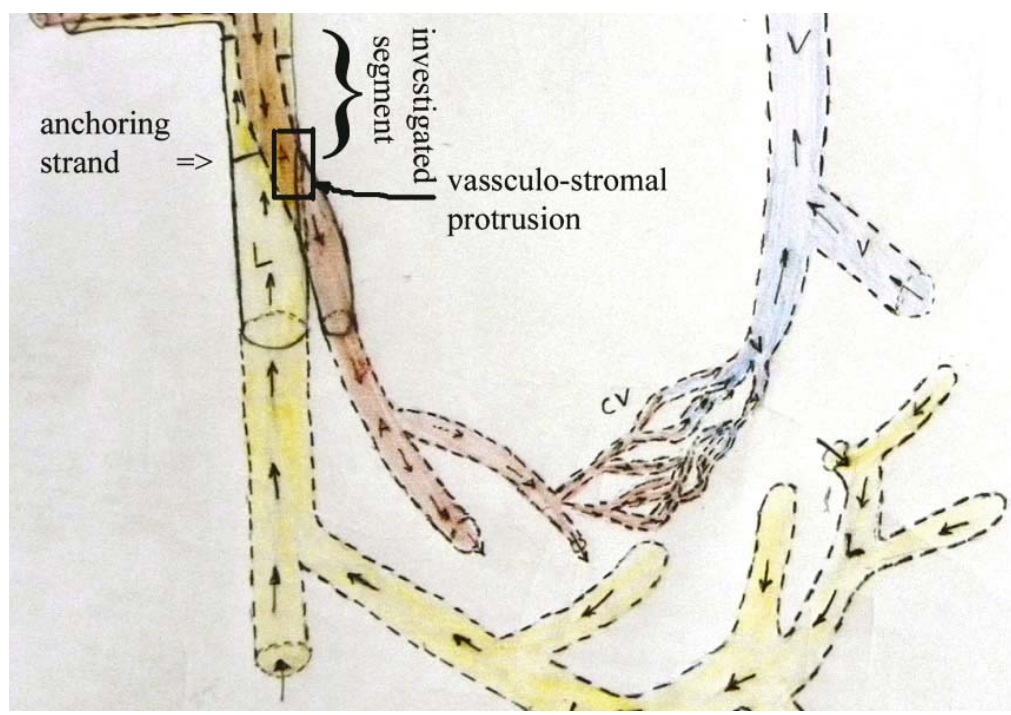

Figure 3. The place of the vessel within the vessel structure in the breast circulation. V - vein; $A$ - artery; CV capillary vessels; arrows designate the direction of blood/lymph flow 
— Ivanov I., et al. Three-dimensional reconstruction of arterial-lymphatic vascular complex...

arterial vessel is situated inside the lymphatic vessel. The presence of valves in the lymphatic vessels [6] supports the unidirectional lymph flow, which is induced by pulsation of the artery, leading to increased pressure within the lumen of the lymphatic vascular segment. The arteries situated inside the pre-collector lymphatic vessels seem to be the pump that sustains stable lymph flow in breast parenchyma.

Another function of the described vascularvascular unit might be related to the vascularstromal protrusion (the initial or terminal part of the vascular-vascular unit) that may act as a dynamic valve [discussed in ref. 3].

The true understanding of the functional properties of these vascular complexes requires

\section{References}

1. Merchant S, Malpica A, Deavers MT, Czapar C, Gershenson D, Silva EG. Vessels within vessels in the myometrium. Am J Surg Pathol. 2002;26(2):232-6.

2. Kutay V, Tuncer M, Harman M, Ekim H, Yakut C.Intracardiac extension of intravenous leiomyoma. Tex Heart Inst J. 2005;32(2):232-4.

3. Ivanov I, Popovska S, Betova T, Baitchev G, Dineva T, Dikova Zl. Immunohisochemical evaluation of the phenomenon "vessels within vessels" in histological specimens from breast parenchyma. Military Medicine 2011;63 (1):1721. [In Bulgarian] functional rather than purely histomorphological studies.

\section{Conclusion}

In conclusion we demonstrated for the first time a $3 \mathrm{D}$ reconstruction of the "vessel-within-thevessel" phenomenon (artery situated inside the pre-collector lymphatic vessel) in the breast of a male subject. We have proved that these vascular complexes are not artificial and they exist in male individuals. The function of those vascular complexes is presently unknown, and a detailed functional evaluation of the described vascular complexes is required.

4. Fiala JC. Reconstruct: a free editor for serial section microscopy. J Microscopy. 2005;218:5261.

5. Sacchi G, Weber E, Agliano M, Raffaelli N, Comparini L. The structure of superficial lymphatics in the human thigh: precollectors. Anat. Rec. 1997;247:53-62.

6. Vankov V, Ovcharov V. Cardiovascular system. In: Vankov V, Ovcharov V, editors. Human anatomy. 10th ed. Sofia: ARSO; 2008. p. 440. [In Bulgarian]

7. Belova N.Cardiovascular system. In: Vitanova L, Garchev R, editors. Human physiology 1st ed. Sofia:ARSO; 2008.p. 104-155. [In Bulgarian] 